\title{
Personalstrategien und Unternehmenskulturen von Unter- nehmen in den neuen Bundesländern - eine Diskussion empirischer Ergebnisse*
}

\author{
Michael Steinhöfel ${ }^{* *}$
}

This article deals with the relations between human ressources strategies and corporate cultures in the New Bundeslaender. It is based on the results of a research project on personnel management within the tranformation from central planned to market economy. The project lasts since 1993. The author discusses the identified types of human ressources strategies and their influence on the development and changes of corporate cultures.

Der Beitrag setzt sich mit den Zusammenhängen zwischen Personalstrategien und Unternehmenskulturen in den neuen Bundesländern auseinander. Er basiert auf Ergebnissen aus einem seit 1993 andauernden Projekt zum Umgang mit dem Personal beim Übergang von der Plan- zur Marktwirtschaft. Der Verfasser stellt die im Forschungsverlauf identifizierten Typen von Personalstrategien dar und diskutiert den Einflu $\beta$ dieser Strategien auf die Entwicklung und Modifizierung von Unternehmenskulturen.

Manuscript received: 19.4.96, revised: 9.5.96, accepted: 13.5.95.

** Michael Steinhöfel, geb. 1957, Dr. oec., Wissenschaftlicher Projektleiter bei der a\&o research GmbH, z.Zt. freigestellt für das DFG-Projekt "Personalstrategien in den neuen Bundesländern nach marktwirtschaftlicher Etablierung der Unternehmen", aktuelle Arbeitsschwerpunkte: Personal- und Organisationsentwicklung, Arbeitsmarkt- und Beschäftigungspolitik. 


\section{Einleitung}

In diesem Beitrag soll diskutiert werden, welcher Zusammenhang zwischen den in Unternehmen der neuen Bundesländer verfolgten Personalstrategien und ihren Unternehmenskulturen besteht bzw. inwieweit die Ausprägungen unterschiedlicher Personalstrategien Folge oder auch Voraussetzung differenzierter Unternehmenskulturentwicklungen sind. Zu diesem Zweck werden die Erhebungsbefunde und Ergebnisse des mehrjährigen Projektes „Personalstrategien in Unternehmen der neuen Bundesländer“ zur Unternehmens- bzw. OrganisationskulturDebatte in Beziehung gesetzt. Damit erfolgt hier eine Konzentration auf die „soft factors“ in den Unternehmen. Die „hard factors“, die im Rahmen der Untersuchungen ebenso erhoben und analysiert wurden, werden in diesem Artikel nicht behandelt, wohl wissend, daß ihre adäquate Gestaltung für die Bewältigung des Wandels und somit für das weitere Überleben der Unternehmen ebenso von Bedeutung sind. Ziel dieses Vorgehens ist es zum einen, die Bedeutung der Arbeits- bzw. Betriebskulturen für die Bewältigung der Metamorphose von planwirtschaftlich organisierten und agierenden Betrieben zu marktwirtschaftlich handelnden Unternehmungen in Ostdeutschland aufzuzeigen. Bei dieser Entwicklung ging es für die Unternehmen vor allem auch, wie es Schein im Zusammenhang mit der Unternehmenskulturdiskussion (Schein 1995:62ff) formuliert, um „Überleben und Anpassung im (transformationsbedingt veränderten - d.A.) äußeren Umfeld“.

Zum anderen soll untersucht werden, inwieweit sich die unternehmensinternen Veränderungen in den erhobenen Personalstrategien widerspiegeln. Werden Personalstrategien in ihrer Wirkung so bewertet, daß ihre Veränderung wesentlich auch das Zusammenwirken von Mensch, Technik und Organisation in einem Unternehmen bestimmt, so werden durch den damit verbundenen Einfluß auf Arbeitshaltungen, Sozialbeziehungen und äußere Arbeitsgestaltung auch Unternehmenskulturen verändert (Neuberger / Kompa 1987:242).

\section{Einige Ausgangsüberlegungen zum Zusammenhang von Personalstrategien und Unternehmenskulturen}

Für den Begriff Unternehmenskultur gibt es heute eine Vielzahl von Definitionen, die aber gemeinsame Kernelemente aufweisen. So beschreiben Kobi und Wüthrich Unternehmenskultur als „die Gesamtheit von geteilten Normen, Wertvorstellungen und Denkhaltungen, die das Verhalten der Mitarbeiter aller Stufen und somit das Erscheinungsbild eines Unternehmens prägen“ (1986:34). Dill versteht darunter „die Grundgesamtheit gemeinsamer Werte, Normen und Einstellungen ..., welche die Entscheidungen, Handlungen und das Verhalten der Organisationsmitglieder“ als „Basiselemente“ prägen (1986:100). Schein definiert in diesem Zusammenhang die Kultur einer Gruppe als „ein Muster gemeinsamer Grundprämissen, das die Gruppe bei der 
Bewältigung ihrer Probleme externer Anpassung und interner Integration erlernt hat, das sich bewährt hat und somit als bindend gilt; und das daher an neue Mitglieder als rational und emotional korrekter Ansatz für den Umgang mit diesen Problemen weitergegeben wird.“ (1995:25). Bei der Bewertung des Zusammenhanges von Personalstrategien und Unternehmenskultur im Zuge der Umwandlung von plan- zu marktwirtschaftlichen Unternehmen folgen wir der Auffassung von Bleicher, für den die Unternehmenskultur ein „Schlüssel für jede erfolgreich zu bewältigende Transformation“ ist (Bleicher 1995). Angelehnt an diese Defintion entsprechen Personalstrategien Mustern im Umgang mit dem Personal; sie sind Handlungsorientierung und zugleich Ausdruck einer vorherrschenden Unternehmenskultur. Sie erwachsen wesentlich aus den Annahmen zu den im Unternehmen geteilten „basic assumptions and beliefs“ (Schein 1985:9) bzgl. des Personals. In dieser Sichtweise sind Personalstrategien impliziter Bestandteil von Unternehmenskulturen.

Der Forschungsansatz zur Erhebung der Personalstrategien und letztlich auch die Interpretation der Befunde wird von der Auffassung geprägt, daß alle Beschäftigtengruppen der verschiedenen Hierarchieebenen und Bereiche eines Unternehmens sowohl Subjekte als auch Adressaten (Objekte) von Personalstrategien sind und dieselbe entsprechend mit ihrem täglichen Handeln im Unternehmen gestalten, umsetzen oder zumindest beeinflussen. Die Einstellungen, die Wertvorstellungen sowie das Verhalten und damit letztlich die Handlungen des Managements und der Mitarbeiter wirken so prägend und sind gleichzeitig Ausdruck der verfolgten Strategien. Die Notwendigkeit eines handlungsorientierten Ansatzes zur Erhebung von Personalstrategien bestätigte sich bei der Realisierung des Projektes, von der Phase der Konstruktion des Erhebungsinstrumentariums bis hin zur Verallgemeinerung der empirischen Befunde. Der mit der Projektrealisierung verbundene Erkenntniszuwachs führte zur mehrfachen Präzisierung und Erweiterung des ursprünglichen Personalstrategie-Begriffes:

„,Personalstrategien sind demnach langfristige, in Wechselbeziehung mit den betrieblichen Reorganisations- und Rationalisierungsstrategien angelegte Konzepte unterschiedlichen Typs, die in differenziertem Maße die Nutzung und Entwicklung der personalen Potentiale der Beschäftigten im Interesse betrieblicher Effizienzsicherung bewirken. Sie werden vom Management konzipiert, von den Mitarbeitern erlebt und reflektiert. Sie beeinflussen das tägliche zielorientierte Handeln aller Akteure im Unternehmen. Personalstrategien entstehen in der Regel iterativ, mit Rückkopplungen und durch permanentes Aushandeln bzw. Konfliktlösung entsprechend den zugrundeliegenden Interessen der betrieblichen Akteure.“ (Denisow et al. 1995:36)

Von dieser Begriffsbestimmung ausgehend, wird zunächst die erste Hypothese formuliert: 
1. Personalstrategien wirken auf die Herausbildung, die Entwicklung und die Veränderung von Unternehmenskulturen, indem sie Anforderungen an Arbeits- und Führungskräfte, Strukturen der Organisation und Kommunikation, Planungs-, Ausführungs- und Kontrollverfahren beeinflussen oder ändern (vgl. auch Neuberger / Kompa, 1987:242). Im „Gegenzug“ sind auch Unternehmenskulturen prägend für Personalstrategien, da sie Ausdruck der im Unternehmen geteilten Auffassungen zur Gestaltung des Zusammenwirkens von Mensch, Technik und Organisation sind.

Dieser dialektische Zusammenhang ist einerseits zu erwarten, da Personalstrategien direkt auf die Beschäftigten eines Unternehmens orientiert sind. Sie beeinflussen zum einen unmittelbar das Individuum, d.h. den Mitarbeiter, seine Sprache, sein Verhalten und seine Gefühle. Zum anderen wirken sie durch ihren Einfluß auf die Organisation der Arbeit vermittelt auf ihn und definieren seine Stellung im Unternehmen. Andererseits können Personalstrategien nicht entgegen der gültigen Grundprämissen und Verhaltensmuster innerhalb eines Unternehmens erfolgreich umgesetzt werden.

Bei der Diskussion der Erhebungsbefunde des Projektes ist gleichzeitig zu berücksichtigen, daß Personalstrategien ebenso wie Unternehmenskulturen auf bereits in der Vergangenheit angelegten und verfolgten Konzepten aufbauen bzw. an diesen anknüpfen und deshalb nur längerfristig wandelbar sind. Nach Staehle (1985:532) entstehen Unternehmenskulturen im Laufe von Jahren oder sogar von Jahrzehnten „durch die allmähliche Verfestigung von bewährten Verhaltensweisen dominanter Organisationsmitglieder“. Diese Grundprämissen und Annahmen, die eine Unternehmenskultur ausmachen, sind Ausdruck von Verhalten der Organisationsmitglieder sowie die Widerspiegelung des sozialen Systems „Unternehmen“. Folgt man dieser Auffassung kann deren Veränderung auch nur in den von Lewin (1947:34) beschriebenen Phasen (Auftauen Verändern - Einfrieren) längerfristig erfolgen. Dieser Verlauf von Wandlungsprozessen wurde auch von Schein (1995:229) explizit für den Kulturwandel in Unternehmen aufgegriffen. Dabei beschreibt er die Möglichkeiten und Inhalte eines Kulturwandels als abhängig vom Entwicklungsstadium des Unternehmens. Die dargelegten Auffassungen zum Wandel von Kulturen sind im vorliegenden Fall in mehrfacher Hinsicht bedeutsam. Zum einen weil die untersuchten Unternehmen eine „Vorgeschichte“ in der DDR hatten und nicht als Unternehmen auf der „grünen Wiese“ gegründet wurden. Die Unternehmen vollzogen den Wandel von alten planwirtschaftlichen zu neuen marktwirtschaftlichen Organisationen, aber in der „alten“ Hülle und mit „alten“ Mitarbeitern. Es gab in den Unternehmen also anerkannte Verhaltensweisen, die sich in der Vergangenheit bewährt hatten, die nun jedoch auf Grund der neuen Anforderungen zu überprüfen waren. Zum anderen sei auch noch darauf verwiesen, daß die Landeskultur der DDR als eine die Arbeitsoder Betriebskulturen (Staehle / Mader 1990, Schreyögg 1996, Stieler-Lorenz 1996) und Personalstrategien prägende Einflußgröße nachwirkt. Das führt auch 
dazu, daß Personalstrategien und damit auch Unternehmenskulturen quasi nicht von heute auf morgen radikal zu verändern waren.

Vor dem dargestellten Hintergund werden zwei weitere Hypothesen aufgestellt:

2. Im Rahmen der Transformation war es praktisch nicht möglich, den Unternehmen in den neuen Bundesländern, „neue“, aus den alten Bundesländern „importierte“ Strategien und Kulturen einfach überzustülpen. Es entstehen Personalstrategien und Unternehmenskulturen , die nicht mit einer Variante typisch „Ost“ oder typisch „West“ zu beschreiben oder zu vergleichen sind.

3. Unternehmen, die (bewußt oder unbewußt) an Erfahrungen und Stärken aus der Vergangenheit anknüpfen (können), bewältigen den Wandel besser als andere Unternehmen.

\section{Die empirische Basis}

Die hier diskutierten Befunde wurden im Rahmen einer Zeitintervallstudie in fünfzehn Unternehmen der neuen Bundesländer erhoben (vgl. Übersicht 1). Die erste Etappe mit zwei Erhebungszeitpunkten in 1993 und 1994 wurde im Dezember 1994 beendet. Die zweite Etappe begann im März 1995 und wird voraussichtlich bis Februar 1997 andauern. ${ }^{2}$

Übersicht 1: Unternehmen nach Branchen, Größenklassen und Regionen

\begin{tabular}{|l|l|l|l|}
\hline Unternehmen & Branche & $\begin{array}{l}\text { Beschäftigten- } \\
\text { größenklasse 1994 } \\
\text { (Personen) }\end{array}$ & Bundesland \\
\hline \hline 1 & Energieversorgung & 1.000 u.m. & (Ost-) Berlin \\
\hline 2 & Elektroanlagenbau & 500 b.u. 1.000 & (Ost-) Berlin \\
\hline 3 & Bürotechnik & 200 b.u. 500 & Thüringen \\
\hline 4 & Elektronik & 10 bis 19 & (Ost-) Berlin \\
\hline 5 & Baugewerbe & 500 b.u. 1.000 & (Ost-) Berlin \\
\hline 6 & Baugewerbe & 500 b.u. 1.000 & (Ost-) Berlin \\
\hline 7 & Baugewerbe & 200 b.u. 500 & Sachsen \\
\hline
\end{tabular}

2 Das Projekt „Personalstrategien und Überlebenschancen von Unternehmen in den neuen Bundesländern“ wurde von der Kommission für die Erforschung des sozialen und Politischen Wandels in den neuen Bundesländern von Dezember 1992 bis Dezember 1994 gefördert. Im Projektteam arbeiteten Dr. Denisow, Karin; Prof. Jacob, Klaus; Prof. StielerLorenz, Brigitte sowie der Autor dieses Beitrages. Die Ergebnisse sind im Projektbericht, veröffentlicht unter dem Titel „Personal im Osten: Anpassungsgröße oder Gestaltungspotential?“, nachzulesen. Die zweite Phase wird als Anschlußprojekt von der Deutschen Forschungsgemeinschaft gefördert. 


\begin{tabular}{|l|l|l|l|}
\hline 8 & Maschinenbau & 200 b.u. 500 & Thüringen \\
\hline 9 & Maschinenbau & 200 b.u. 500 & (Ost-) Berlin \\
\hline 10 & Nahrung/Genuß & 200 b.u. 500 & Sachsen-Anhalt \\
\hline 11 & Nahrung/Genuß & 100 b.u. 200 & (Ost-) Berlin \\
\hline 12 & Nahrung/Genuß & 50 b.u. 100 & Brandenburg \\
\hline 13 & Holzverarbeitung & 50 b.u. 100 & Sachsen \\
\hline 14 & Energieversorgung & 1.000 u.m. & Brandenburg \\
\hline 15 & $\begin{array}{l}\text { Monetäre Dienst- } \\
\text { leistungen }\end{array}$ & Sachsen \\
\hline
\end{tabular}

Zur Projektrealisierung wurden qualitative Methoden der Sozialforschung, insbesondere der Arbeitspsychologie und der Industriesoziologie, eingesetzt. Dieses Herangehen wird durch einen interdisziplinären Ansatz geprägt, der vor allem auf Grund der heterogenen Zusammensetzung des Forschungsteams realisiert werden kann. Es kamen, neben der Dokumentenanalyse und Beobachtungen, insbesondere strukturierte Interviews und Gruppendiskussionen zum Einsatz. ${ }^{3}$ Bei diesem Herangehen wird die Forschung, wie von Ochsenbauer/Klofat (1987:99) beschrieben, letztlich zur gemeinsamen „Aktion von Analysierenden (Forscher, Berater) und Analysierten (Mitarbeiter eines Unternehmens)“. Mit dem Ansatz bei der Erhebung von Personalstrategien wird somit auch der Forderung an die Unternehmenskulturforschung entsprochen, qualitative, hermeneutisch orientierte und gegenstandsangemessene Verfahren zum Einsatz zu bringen (v. Rosenstiel 1993:19). Es werden Daten zu Sachverhalten, Verhalten und Interaktionen erhoben, die mit Methoden der quantitativen empirischen Sozialwissenschaft nicht zugänglich sind. Qualitative Methoden sind somit geeignet, die Unternehmung als von handelnden Menschen abhängige Organisationen zu begreifen und zu erfassen. Die so gewonnenen Erkenntnisse werden in der Diskusssion mit den beteiligten Mitarbeitern aus den Unternehmen interpretiert und „kommunikativ validiert“ (vgl. Scheele 1991:276).

Entsprechend dem handlungsorientierten Forschungsansatz erfolgte zur Auswertung der erhobenen Befunde die Zuordnung der gebildeten Analysekategorien zu personalstrategischen Handlungsfeldern (vgl. Übersicht 2). Die unterschiedlichen Ausprägungen der Analysekategorien und damit der

3 In der ersten Untersuchungswelle im Sommer 1993 fanden in den fünfzehn Unternehmen 200 Einzelinterviews statt. Dabei wurden Vertreter des Managements, Angestellte und gewerbliche Mitarbeiter sowie Mitglieder der Betriebsräte befragt. Diese Einbeziehung der Vertreter verschiedener Hierarchieebenen und Beschäftigtengruppen wurde auch in der zweiten Untersuchungswelle im Herbst 1994 gesichert. Dazu wurden in elf Unternehmen 34 Gruppengespräche und 31 Einzelinterviews geführt 
Handlungsfelder, der Klassifizierungsmerkmale, bilden die Grundlage für die Typisierung der verfolgten Personalstrategien.

Die verwendeten Analysekategorien erwiesen sich ebenso geeignet, wesentliche Teile von Unternehmenskulturen zu erfassen, da sowohl die Elemente der materiellen Ebene, wie Arbeitsaufgaben, ihre Unterteilung und Verknüpfung, die eingesetzte Technik, die Führungsstrukturen und -instrumente u.a. als auch deren konkrete Ausgestaltung auf Basis der vorherrschenden Annahmen, Wertvorstellungen und Überzeugungen (Baitsch 1996) erhoben werden konnten.

\section{Ergebnisse}

Nachfolgend werden ausgewählte Untersuchungsergebnisse präsentiert. Dabei erfolgt im ersten Teil die Darstellung der unterschiedlichen Typen von Personalstrategien, die nach Auswertung des erhobenen Datenmaterials identifiziert werden konnten. Diese Ausführungen dienen auch der kurzen Darstellung und dem Nachweis des hypothetisch angenommenen, dialektischen Zusammenhanges zwischen Personalstrategien und Unternehmenskulturen (Hypothese 1). Im Anschluß daran werden die weiteren in Abschnitt 2 formulierten Hypothesen anhand der erhobenen Befunde zusammenfassend diskutiert.

\subsection{Zur Typisierung von Personalstrategien}

Im Ergebnis unserer Untersuchungen war es möglich, die Personalstrategien auf Grund der vorgefundenen unterschiedlichen Ausprägungen der personalstrategischen Handlungsfelder wie folgt idealtypisch herauszuarbeiten (vgl. Abb.1):

A) qualifikations-, motivations- und kompetenzorientierte Personalstrategien,

B) technikorientierte Personalstrategien und

C) Strategien der strukturellen Personalanpassung.

Die unterschiedlichen Strategien im Umgang mit dem Personal waren vor allem im Ergebnis der zeitlichen Entwicklung in den Unternehmen stärker erkennbar. Im Rahmen der zweiten Erhebung 1994 konnte eine weitere Ausprägung bzw. Vertiefung von Ansätzen zur Differenzierung der Personalstrategien zwischen den Unternehmen festgestellt werden. Die vorgenommene Typisierung von Personalstrategien ist nicht als Bewertung im Sinne von schwarz/weiß, gut/schlecht, falsch/richtig oder besser/schlechter und damit als Empfehlung für das praktische Handeln der Akteure aufzufassen (vgl. Lösch 1993:356). Vielmehr sind die unterschiedlichen Personalstrategietypen im Zusammenhang mit spezifischen Faktoren und Optionen (Rahmenbedingungen) sowie ihrer differenzierten Wahrnahme durch alle handelnden Personen entstanden. Dabei werden Rahmenbedingungen nicht als die Ursachen des Handelns der Akteure sondern als Grenzen definierende Bedingungen betrachtet (Lang/Alt 1995:358). Die differenzierten Personalstrategien in den untersuchten Unternehmen waren 
dabei bisher offensichtlich geeignet, daß Überleben der Unternehmen beim Übergang von der Plan- zur Marktwirtschaft zu sichern.

Übersicht 2: Handlungsfelder und Analysekategorien zur Erhebung von Personalstrategien

\begin{tabular}{|l|l|}
\hline Handlungsfelder & Analysekategorien \\
\hline \hline $\begin{array}{l}\text { Unternehmens- und Orga- } \\
\text { nisationsentwicklung }\end{array}$ & $\begin{array}{l}\text { Unternehmensentwicklung; } \\
\text { Ausgliederung/Hauptfunktionen; } \\
\text { Hierarchisierung/Aufbau der Organisation; }\end{array}$ \\
\hline $\begin{array}{l}\text { Arbeitsanforderungen/ } \\
\text { Arbeitsorganisation }\end{array}$ & $\begin{array}{l}\text { Arbeitsaufgabe; Qualifikationspotential; Beanspruchung; } \\
\text { Technisierung; Arbeitsorganisation; } \\
\text { Produkte; Flexibilitätsanforderungen; } \\
\text { Arbeitszeit; }\end{array}$ \\
\hline Personalabbau & $\begin{array}{l}\text { Personalstatistik; } \\
\text { Personalstrukturen; } \\
\text { Wege des Personalabbaus; }\end{array}$ \\
\hline Personalentwicklung & $\begin{array}{l}\text { Personalplanung; Personalentwicklung; } \\
\text { Personalbeurteilung; Personalstrukturen; }\end{array}$ \\
\hline Personalrekrutierung & $\begin{array}{l}\text { Personalplanung; } \\
\text { Qualifikationspotential; }\end{array}$ \\
\hline Personalführung & $\begin{array}{l}\text { Führung; } \\
\text { Hierarchisierung; } \\
\text { Geschäftsführungs- Mitarbeiter-Verhältnis; }\end{array}$ \\
\hline Personalmotivation & $\begin{array}{l}\text { Motivation; } \\
\text { Lohn-Leistung- Bewertung; } \\
\text { Arbeitszeit; } \\
\text { Personalbeurteilung; }\end{array}$ \\
\hline Partizipation & $\begin{array}{l}\text { Information; } \\
\text { Verbesserungswesen; Einbeziehung in Veränderungsprozesse; } \\
\text { Unternehmerische Verantwortung; }\end{array}$ \\
\hline Personalarbeit & $\begin{array}{l}\text { Zusammenarbeit BR mit anderen betrieblichen Akteuren; } \\
\text { Interessenkonflikte; } \\
\text { Aufgabenfelder: }\end{array}$ \\
\hline $\begin{array}{l}\text { Stellung der PA; } \\
\text { Aufgaben bei Personalarbeit; } \\
\text { Arbeitsbewertung; }\end{array}$ \\
\hline
\end{tabular}

Die Unterschiede, die letztlich zur Typisierung führten, liegen im Herangehen der Unternehmen an die Bewältigung der neuen Anforderungen im Rahmen der Systemtransformation und den veränderten Wettbewerbsbedingungen 
begründet. Sie wurden durch die differenzierte Bewertung von Rolle und Stellung des Personals in der Unternehmensentwicklung hervorgerufen, die wiederum von vielfältigen objektiven und subjektiven Ursachenkomplexen, wie u. a. Sozialisation und Menschenbild, Produktionskonzepte, Fertigungsart und Technologieeinsatz, Unternehmensgröße und Organisationsstruktur, Führungsverhalten und Führungserleben, und ihrem unterschiedlichem Zusammenwirken geprägt ist. Diese Unterschiede prägen so über die verfolgten Personalstrategien auch die Unternehmenskulturen. Zukünftig wird zu untersuchen sein, inwieweit die diesen Unternehmenskulturen zugrunde liegenden „basic assumptions and beliefs“ (Schein 1995) dauerhaft geteilt, akzeptiert oder auch nur vorläufig hingenommen werden bzw. ob und wie die zunehmend „frei am Markt“ agierenden Unternehmen unter steigendem Wettbewerbsdruck mit Veränderungen in den Personalstrategien und darüber vermittelt in der Unternehmenskulturentwicklung reagieren. Die betrieblichen Kultur ist zugleich Ausgangspunkt bzw. Option für die angestrebten Strategien.

Die Personalstrategien werden hier verallgemeinernd und idealtypisch dargestellt. In den Unternehmen waren innerhalb der einzelnen Typen wiederum differenzierte Entwicklungen in einzelnen Handlungsfeldern nachweisbar, jedoch war übergreifend eine bestimmte Grundrichtung in der Gestaltung der Mensch-Technik-Organisations-Beziehungen in der Projektphase bis 1994 prägend. Bei der Einschätzung der unterschiedlichen Personalstrategien ist zu beachten, daß „reine“ und hundertprozentig abgrenzbare Typen, wie sie nachfolgend dargestellt werden, in der Praxis nicht vorkommen, sondern daß die Übergänge zwischen den Gruppen fließend sind. Gleichzeitig ist zu berücksichtigen, daß die unterschiedlichen Strategien auf Grund der übergreifenden Anforderungen und der exogen gesetzten Rahmenbedingungen der Systemtransformation eine Reihe von Gemeinsamkeiten aufweisen, auf deren Darstellung hier aber verzichtet wird. (vgl. Denisow et al. 1995:111ff). Für die Zukunft bedeutet das, daß längerfristig sowohl eine weitere Modifikation der dargestellten Typen, aber auch der Übergang von einem zum anderen Personalstrategietyp längerfristig erwartet werden kann.

Die vorhandenen, geteilten weil bewährten Muster zur Bewältigung externer und innerbetrieblicher Probleme wirken auch bei der Konzipierung, Umsetzung und Widerspiegelung der Personalstrategie eines Unternehmens. Personalstrategien sind auf Dauer nur durchsetzbar und ihr Erfolg ist davon abhängig, ob und inwieweit sie von betrieblichen Grundprämissen und Verhaltensmustern und damit von der Entwicklung ihrer Unternehmenskultur ausgehen. 
Abbildung 1: Schematische Darstellung der Typisierung von Personalstrategien nach der Grundrichtung der Ressourcennutzung bei der Gestaltung der Mensch-Technik-Organisations-Beziehungen

Qualifikations-, motivations- und kompetenzorientierte Strategie

Technik

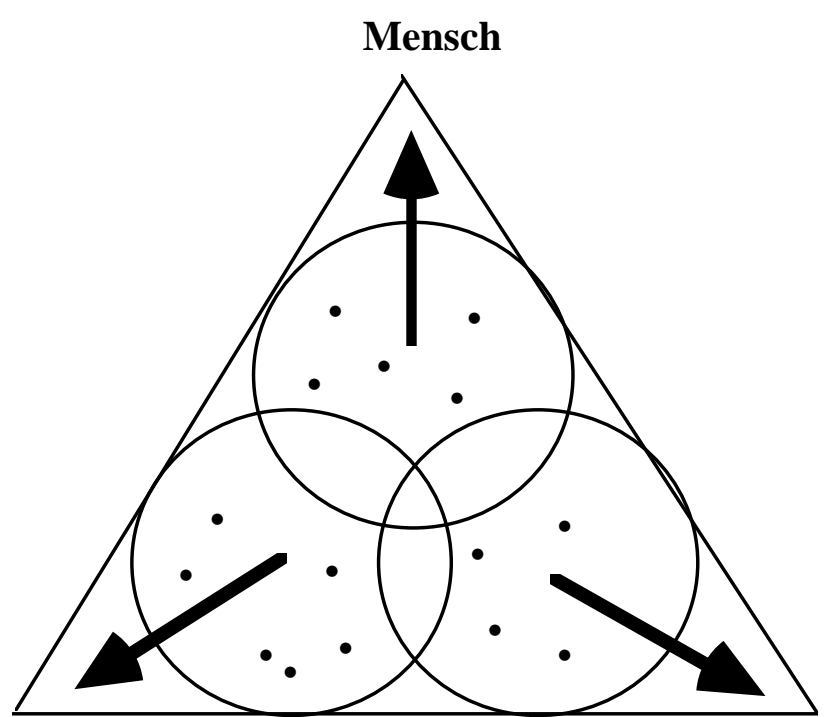

Technikorientierte

Strategie der strukturellen

Personalstrategie

Personalanpassung

• = Unternehmen

\section{Typ A: Qualifikations-, motivations- und kompetenzorientierte Personal- strategie - Die Arbeitskraft wird als Gestaltungspotential aufgefaßt.}

Bei diesem Typ, den wir in fünf der untersuchten Unternehmen feststellen konnten, bildet deshalb das vorhandene und weiter - vor allem qualitativ - auszugestaltende Arbeitskräftepotential einen wichtigen Ausgangspunkt betrieblicher Sachentscheidungen. In der Potentialnutzung und -entwicklung wird eine entscheidende Basis für die Entwicklung des Unternehmens und für wirtschaftliche Erfolge gesehen. Vom Menschenbild her wird der sich selbstverwirklichende Mitarbeiter gefördert und gefordert. Charakteristisch ist auch, daß das vorhandene Humanpotential zu einem wichtigen Ausgangspunkt für konzeptionelle Entscheidungen über die weitere Unternehmensentwicklung, speziell die Erzeugnisentwicklung, gemacht wurde. Die betrieblichen Führungs-, Motivations- und Personalentwicklungskonzepte sind auf den Erhalt, die Erschließung und die Entwicklung der Leistungs- und Qualifikationspotentiale der Mitarbeiter gerichtet. Die Handlungsweise ist prospektiv und perspektivisch 
angelegt. Die Beschäftigten werden in Ansätzen in die betrieblichen Gestaltungsprozesse einbezogen. Management und Mitarbeiter teilen in diesen Unternehmen gemeinsame Auffassungen bzgl. des Zusammenwirkens von Mensch, Technik und Organisation. Für die Unternehmenskultur sind hier u.a. ausgeprägte, auf Interaktion ausgerichtete Informations- und Kommunikationsbeziehungen, die Partizipation der Mitarbeiter an der Unternehmensentwicklung sowie die Identifikation der Mitarbeiter mit dem Unternehmen kennzeichnend.

\section{Typ B: Technikorientierte Personalstrategie - Die Arbeitskraft ist Anpas- sungsgröße.}

Dieser Strategietyp, dem wir sechs Unternehmen aus unserem Sample zuordneten, geht von einer Adaptation des quantitativen und qualitativen Arbeitskräftepotentials an die veränderten Anforderungen, speziell der technischen Entwicklung, aus. Die Arbeitskräfte sind dabei eher eine „Manövriermasse“ bei betrieblichen Entscheidungen. Personalentwicklung findet nur insofern statt, als sie zum Inganghalten der Technik und der Prozesse unabdingbar ist. Es handelt sich dabei um ein reaktives, kaum perspektivisch orientiertes Verhalten, z.B. werden Qualifikationsdefizite zum Ausgang betrieblicher Weiterbildung genommen. Technikorientierte Personalstrategie bedeutet, daß mit personalen Prozessen, wie Abbau oder Neueinstellung, Entwicklung, Einsatz, Integration u.a.m. auf die Herausforderungen der Umsatzentwicklung, der Produkt- und Prozeßinnovation, vor allem aber der technisch-technologischen Entwicklung reagiert wird. Der Kostenaspekt determiniert das Herangehen. Im Blickpunkt des Managements steht hier kaum die Frage, welche Potentiale im vorhandenen Personal liegen und wie diese für die Effektivierung der laufenden Arbeit oder auch für neue betriebliche Handlungsfelder genutzt und weiterentwickelt werden können. Anpassung heißt aber auch, daß das Management auf die Einordnung des Verhaltens der Mitarbeiter an die von ihm vorgegebenen Prinzipien und Regeln besteht. Ausgangspunkt für das Handeln ist überwiegend die Sichtweise auf die Mitarbeiter als „economic men“. Dieses Vorgehen wird aus Sicht der Mitarbeiter auf Grund der fehlenden Alternativen eher hingenommen den akzeptiert oder gar geteilt.

Die Überlebenserwartungen und damit auch die Kultur im Unternehmen sind davon geprägt, daß das Bemühen um Mitarbeitermotivation und -partizipation, um Erschließung von Innovationspotentialen der Fachkräfte sowie um stimulierende Führungsstile nicht als Weg zur Optimierung des Zusammenwirkens von Mensch-technik-Organisation gewählt wurde. Vielmehr werden die Unternehmenschancen (noch) isoliert in der Nutzung moderner Technik gesehen. 


\section{Typ C: Strategie struktureller Personalanpassung - Die Arbeitskraft ist hier ebenfalls Anpassungsgröße.}

Dieser in sich widersprüchliche Typ, der für vier Unternehmen charakteristisch ist, kann durch folgende Merkmale charakterisiert werden: Rationeller Personaleinsatz sowie Personalabbau sollen vorrangig durch die Organisationsgestaltung erreicht werden, wobei den verbleibenden Mitarbeitern expertokratisch und durch Managemententscheidungen veränderte Aufgabenfelder zugewiesen werden. Diese Aufgabenfelder werden in der Tendenz durch „Personalausdünnung“ und „Verschlankung“ geprägt. Veränderungen in den Arbeitsaufgaben und Arbeitsinhalten werden unter Rationalisierungsaspekten vorgenommen. Die Mitarbeiter haben wenig oder gar keine Möglichkeit der Mitwirkung bei der Auswahl und Einführung alternativer Organisationsformen und bei Entscheidungsprozessen. Die Mitarbeiter haben eher zu reagieren und zu akzeptieren. Es existieren stringente Abhängigkeiten der Mitarbeiter von Vorgesetzten-Entscheidungen in festgefügten Hierarchien. Die Unternehmenskultur wird hier durch die vorgegebenen Organisationsstrukturen stark geprägt, die damit ihrerseits Möglichkeiten und Grenzen der Entfaltung der Mitarbeiter setzen.

\subsection{Diskussion der Hypothesen zu Stabilität und Veränderung als prägende Aspekte von Personalstrategien und Unternehmenskulturen}

Bei der Betrachtung von Entwicklung und Veränderung der Unternehmenskulturen in den neuen Bundesländern ist zu bedenken, daß die Veränderungsprozesse in den Unternehmen nicht isoliert abliefen. Vielmehr fanden und finden alle betrieblichen Umgestaltungen auch unter dem Eindruck und Einfluß von den durch die Belegschaften erlebten und reflektierten Veränderungen sowohl im gesellschaftlichen als auch im familialen Umfeld statt und sind in ihrer Wechselwirkung auf betriebliche Prozesse zu berücksichtigen. Nach fünf Jahren Transformation sind Unternehmenskulturen im Entstehen, die sich nicht nur von den Arbeits- oder Betriebskulturen aus der ehemaligen DDR sondern auch von Unternehmenskulturen aus den alten Bundesländern unterscheiden. Unsere Untersuchungen belegen die Hypothese, daß es praktisch nicht möglich war, den Unternehmen in den neuen Bundesländern, „neue“, aus den alten Bundesländern „,importierte“ Strategien und Kulturen einfach überzustülpen. Vielmehr wirken die in der Vergangenheit gelebten Werte, Haltungen, Einstellungen und Verhaltensweisen unter den neuen Bedingungen modifizierend. Es entstehen offensichtlich Personalstrategien und Unternehmenskulturen, die nicht mit einer Variante typisch „Ost“ oder typisch „West“ zu beschreiben oder zu vergleichen sind. Es bilden sich „Lokale Theorien“ in den Unternehmen der neuen Bundesländer heraus, die Ausdruck der im Unternehmen vorherrschenden, von den Akteuren aus ihren jeweiligen bisherigen Erfahrungen entwickelten und durch das Erleben neuer Rahmenbedingungen veränderten Menschenbilder, Technikkonzepte und Überzeugungen hinsichtlich angemessener 
Organisationsformen sind (Baitsch 1996). Sie repräsentieren damit diejenigen organisationsbezogenen Schemata, die in Interaktionsbeziehungen erworben, verfestigt und verändert sowie von einer Mehrzahl der Mitglieder des Unternehmens geteilt werden (Hofbauer 1991).

Das soll kurz am Beispiel des betrieblichen Vorschlagswesens, daß als Teil des personalstrategischen Handlungsfeldes Partizipation (vgl. Übersicht 2) erhoben wurde, verdeutlicht werden: Nachdem mit der Wende in der Mehrheit der Unternehmen das politisch überfrachete und belastete Neuererwesen abgeschafft wurde, oder auch einfach einschlief, konnte im Rahmen der zweiten Erhebungswelle 1994 Versuche zur Einführung institutionalisierter Formen des Vorschlagswesens, wie sie in den alten Bundesländern praktiziert werden, identifiziert werden. Sie werden jedoch von den Beschäftigten in den Unternehmen kaum und nur zögerlich angenommen. Dabei sind zwei Bewertungsmuster erkennbar: Erstens: Es wird postuliert, daß Verbesserungsvorschläge als Bestandteil der Arbeitsaufgabe angesehen und direkt in die Arbeitsprozesse eingebracht werden. Zweitens: Institutionalisierte Formen werden offen abgelehnt, weil bei diesem Vorgehen negative Erfahrungen und sinnlose „Verbesserungen“ erlebt wurden. Hier stehen die Unternehmen, wenn sie wirksam das „Gold aus den Köpfen“ ihrer Mitarbeiter schürfen wollen, vor der Aufgabe, neue, ansprechende, möglichst unbürokratische Formen für das Vorschlagswesen zu entwickeln, die bisher weder in den Unternehmen der DDR noch in denen der „alten“ BRD praktiziert wurden.

Die Entwicklung von Personalstrategien und Unternehmenskulturen unterliegt in den ostdeutschen Unternehmen spezifischen Bedingungen, die zum einen aus der Vergangenheit in einem anderen Gesellschaftssystem und zum anderen aus der exogen induzierten Transformation herrühren. Der in den untersuchten Unternehmen erfolgte gravierende Personalabbau hat stark reduzierte Belegschaften hervorgebracht. In unserem Sample wurden diese auf bis zu 10\% der ursprünglichen Stärke reduziert. Damit verbunden war u.a.:

- die Erhöhung des Anteils der gewerblichen Mitarbeiter zuungunsten der Angestelltenbereiche,

- der Anstieg des durchschnittlichen formalen Qualifkationsniveaus infolge der Veränderung der Qualifikationsstruktur zugunsten der Höherqualifizierten und

- die Erhöhung des Durchschnittsalters der Beschäftigten durch Abbau jüngerer und über 55jähriger Mitarbeiter mit der Folge der Konzentration von Beschäftigten in der Altersgruppe zwischen 40 und 55 Jahren. ${ }^{4}$

Es sind somit infolge der Abbauprozesse Belegschaften entstanden, die über eine lange gemeinsame Betriebszugehörigkeit von z.T. mehr als 20 Jahren 
verfügen und über lange Zeit eine gemeinsame Kultur erlebt, z. T. geprägt und z.T., bewußt oder unbewußt, auch bewahrt haben. Die Sozialisation der überwiegenden Anzahl der Arbeitnehmer, aber auch des Managements, bildete somit eine wichtige Einflußgröße auf die Herausbildung, Entwicklung, Umsetzung und Akzeptanz von Personalstrategien und Unternehmenskulturen. Dabei bestand von Anfang an zwischen den Akteuren eine starke Konsensorientierung, die den Erhalt des Unternehmens als Voraussetzung für den Erhalt des Arbeitsplatzes in den Vordergrund rückte. Diese das Handeln zumindest in der ersten Phase bis Ende 1993 stark prägende Konsensorientierung resultierte aus der verbindenden Herkunft, dem in der DDR ausgeprägten Kollektivgefühl und den häufig gleichen Befürchtungen hinsichtlich der Zukunftssicherung. Allerdings ist $\mathrm{zu}$ erwarten, daß perspektivisch die aus der DDR-Vergangenheit herrührenden Sozialisationseffekte durch die neu gemachten gesellschaftlichen Erfahrungen mehr und mehr modifiziert werden.

Die Ergebnisse der Untersuchungen bestätigen die Hypothese, daß gerade die Unternehmen, die auf ,alte Stärken“ also auch auf eine gewisse Stabilität setzen konnten und gesetzt haben, die erste Phase der Transformation relativ gut bewältigt haben. Als „alte Stärken“ werden in diesem Zusammenhang vorhandene Werte, wie Zusammengehörigkeitsgefühl und Betriebsverbundenheit, Hilfsbereitschaft, die vorhandenen Qualifikationen, lange bestehende Kooperations- und Kommunikationsbeziehungen etc. bezeichnet. Diese Stärken sollen kurz am Beispiel von Qualifikation, Betriebsverbundenheit und gruppenorientiertem Denken vermittelt werden.

Im Zusammenhang mit der Bedeutung der vorhandenen Qualifikation für die Bewältigung der Transformation wird nicht nur die eingebrachte Qualifikation als Stärke bewertet. Noch größerer Stellenwert wird der immensen Lernbereitschaft und der Fähigkeit zur selbständigen Wissensaneignung eingeräumt. Ohne diese Bereitschaft und Fähigkeiten sowie ohne den Anspruch der einzelnen Mitarbeiter, „es packen zu wollen“, wäre es gar nicht möglich gewesen, die Veränderungen im Unternehmen und persönlich-privaten Bereich parallel $\mathrm{zu}$ den quantitativ und oft auch qualitativ gestiegenen Arbeitsanforderungen zu bewältigen. Im Zusammenhang mit der Betriebsverbundenheit zeigte sich in zahlreichen Gesprächen, daß selbst Mitarbeiter, die bereits den Zeitpunkt ihrer Entlassung kannten, sich trotzdem für „ihr“ Unternehmen bis zum letzten Tag engagierten. Sie wollten trotz des bevorstehenden Endes der Betriebszugehörigkeit noch einen Beitrag für das Überleben „ihres“ Unternehmens leisten. Die Nutzung der Teamorientierung ostdeutscher Mitarbeiter erleichterte den Unternehmen die Bewältigung der fast täglichen neuen und hohen Anforderungen. Dabei differenzierten die Mitarbeiter schnell selbst in Bewahrenswertes (soziale Bezüge in der Gruppe, Kommunikation, gegenseitige Hilfe und Unterstützung) und Hinderliches (Leistungszurückhaltung, Verweigerung von Verantwortungswahrnahme, informelle Bündnisse 
gegen Vorgesetzte). Dieser Rückgriff auf das Vorhandene bedeutete somit nicht, daß sich die Unternehmen und die Mitarbeiter den bestehenden Reorganisationsanforderungen verschlossen haben. Vielmehr erfolgte ein Mix von Altem und Neuem, der die Unternehmen in die Phase der weiteren Stabilisierung begleitete. Dieses Anknüpfen an „alte Stärken“ konnten wir nicht nur in Unternehmen mit „reinem“ Management aus den neuen Bundesländern feststellen. Vielmehr haben auch Führungskräfte aus den alten Bundesländern daran angeknüpft, sofern sie sich der Zusammenhänge von Personalstrategien und Unternehmenskulturen sowie deren Zählebigkeit und Widerstandsfähigkeit gegen abrupte Veränderungen aus ihrer Arbeit bewußt waren.

Es bleibt aber auch festzuhalten, daß die Unternehmen in der Transformation auch mit hemmenden oder behindernden Einflußfaktoren, wie z.B. starkes Sicherheitsdenken, Inaktivität und Abwartehaltungen der Mitarbeiter, konfrontiert waren. Das Tempo und der Grad ihrer Überwindung beeinflußte das weitere Überleben der Unternehmen wesentlich. Dabei war erkennbar, daß die Unternehmen, die menschzentrierte Modernisierungskonzepte bzw. Strategien verfolgten, schneller diese eher hemmenden Faktoren überwinden und die Mitarbeiter in die Unternehmensentwicklung einbeziehen konnten.

Ein weiterer Aspekt hat bei der Gestaltung, dem Wandel bzw. der Veränderung von Personalstrategien und Unternehmenskulturen prägend gewirkt und ist bei der Interpretation der Befunde zu berücksichtigen: Die aus der Transformation resultierenden und die parallel dazu sich verschärfenden neuen wettbewerblichen Anforderungen haben in den Unternehmen, sowohl bei den Mitarbeitern als auch beim Management, unabhängig von ihrer geographischen Herkunft, umfangreiche Lern- und Qualifizierungsprozesse - primär in der Arbeit selbst - induziert. Diese waren gleichzeitig mit der Evaluierung der vorhandenen mit eingebrachten Erfahrungen, Kenntnisse, Verhaltensweisen bis hin zur Sprache verbunden waren und sind. Dabei sind spezifische Formen der Kompetenzentwicklung, vor allem durch die Verbindung von Lernen und organisationaler Entwicklung entstanden, die von prinzipieller Bedeutung für die qualitative Entwicklung betrieblicher Weiterbildung sein können (vgl. Fischer / Jacob 1995). Für die Zukunft wird in diesem Zusammenhang zu prüfen sein, ob und inwieweit aus einzelnen Ansprüchen der Kompetenzorientierung unternehmensübergreifende Kultur werden kann bzw. wird.

Die über das personalstrategische Handeln der Akteure in den Unternehmen der neuen Bundesländer sich vollziehenden und nachweisbaren Wechselbeziehungen zwischen Personalstrategie- und Unternehmenskulturentwicklung werden bei der Etablierung der Unternehmen in der Marktwirtschaft weitere Modifizierungen erfahren. Das ist ursächlich auf die mit wachsenden nationalen und internationalen Herausforderungen verbundenen wirtschaftlichen Turbulenzen zurückzuführen. Die sozialwissenschaftliche Begleitung der Unternehmen bis 1997 gibt die 
Möglichkeit, diese Entwicklung zu dokumentieren und die daraus gewonnen Erkenntnisse für die Theorie und Praxis der Personalstrategie- und Unternehmenskulturentwicklung nutzbar zu machen.

\section{Literatur}

Baitsch, C. (1996): Unternehmenskultur und Transformationsprozesse - ein Interpretationsversuch. In: Lang, R. (Hrsg.): Wandel von Unternehmenskulturen in Ostdeutschland und Osteuropa. München / Mering. S. 257-270.

Baitsch, C. (1993): Was bewegt Organisationen. Frankfurt / New York.

Blickle, G. / Müller, G.F. (1994): Lean Management - zwischen Bekehrungseuphorie und Skepsis. In: management revue 1/94. S. 5-15.

Bleicher, K. (1995): Kommentar auf dem Einband. In: Schein, E.: Unternehmenskultur. Frankfurt/New York.

Birnbaum, H. / Kornbichler, T. (1994): Transformation ostdeutscher Arbeitskultur beim Übergang zu einer Organisationskultur marktwirtschaftlicher Prägung. In: Kornbichler, T. I Hartwig, C.-J.: Kommunikationskultur und Arbeitswelt. S. 231 - 239.

Deal, T.E. / Kennedy, A.A. (1987): Unternehmenserfolg durch Unternehmenskultur. Bonn.

Denisow, K. / Jacob, K. / Steinhöfel, M. / Stieler-Lorenz, B. (1995): Personal im Osten: Anpassungsgröße oder Gestaltungspotential? Personalstrategien und Überlebens-chancen von Unternehmen in den neuen Bundesländern. Berlin.

Dill, P. (1986): Unternehmenskultur. München.

Fischer, E. / Jacob, K. (1995): Analyse der Entwicklung der nahrungs- und Genußmittelbranche unter besonderer Berücksichtigung des Weiterbildungsaspektes. Berlin.

Hofbauer, W. (1991): Organisationskultur und Unternehmenstrategie. München.

Kobi, J.-M. / Wüthrich, H.A. (1986): Unternehmenskultur verstehen, erfassen und gestalten. Landsberg / Lech.

Lang, R. / Alt, R. (1995): Handlungsspielräume des ostdeutschen Managements im Umbruch. In: Sadowski, D. / Czap, H. / Wächter, H.: Regulierung und Unternehmenspolitik. Methoden und Ergebnisse der betriebsiwrtschaftlichen Rechtsanalyse. S. 355-377.

Lewin, K. (1947): Frontiers in Group Dynamics. New York.

Lösch, D. (1993): Der Weg zur Marktwirtschaft: Grundzüge einer Theorie der Transformationspolitik. Baden-Baden.

Mader, N. / Staehle, W. (1991): Arbeitskultur in der ehemaligen DDR: Ein Pendant zur Unternehmenskultur. Berlin.

Neuberger, O. / Kompa, A. (1987): Wir, die Firma. Weinheim.

* Für die zahlreichen Hinweise und die hilfreiche Diskussion im Verlaufe des Entstehungsprozesses dieses Beitrages möchte ich ganz herzlich Prof. Dr. Brigitte StielerLorenz und Dipl.psych. Frank Glowitz (a\&o research GmbH Berlin) sowie Prof. Dr. Rainhart Lang (TU Chemnitz-Zwickau) danken. 
Ochsenbauer, C. / Klofat, B. (1987): Überlegungen zur paradigmatischen Dimension der aktuellen Unternehmenskulturdiskussion in der Betriebswirtschaftslehre. In: Heinen, E.: Unternehmenskultur. München. S. 67 - 106.

Rosenstiel, L.v. (1993): Unternehmenskultur - einige einführende Anmerkungen. In: Dierkes, M. / Rosenstiel, L.v. / Steger, U. (1993): Unternehmenskultur in Theorie und Praxis. Frankfurt / New York. S. 8-22.

Scheele (1991): Dialogische Hermeneutik. In: Flick, U. et al. (Hrsg.): Handbuch Qualitative Sozialforschung: Grundlagen, Konzepte, Methoden und Anwendungen. München. S. 274-278.

Schein, E.H. (1985): Organizational Culture and leadership. San Francisco/London.

Schein, E.H. (1995): Unternehmenskultur. Frankfurt/New York.

Schreyögg, G. (1996): Zum Verhältnis von Landeskultur und Unternehmenskultur. In: Lang, R. (Hrsg.): Wandel von Unternehmenskulturen in Ostdeutschland und Osteuropa. München / Mering. S. 65-72.

Staehle, W. (1985): Management. München.

Steinhöfel , M. / Stieler, B. / Tügel, A. (1993): Veränderung betrieblicher Personalstrukturen im Transformationsprozeß. Halle/S.

Stieler-Lorenz, B. (1996): Wandel der Unternehmenskulturen in Ostdeutschland. In: Lang, R. (Hrsg.): Wandel von Unternehmenskulturen in Ostdeutschland und Osteuropa. München / Mering. S. 107-120.

Ulich, E. (1991): Arbeitspsychologie. Zürich. 